\title{
CONSUMO DE MÉTODOS CONTRACEPTIVOS PELA POPULAÇÃO DO MUNICÍPIO DE SÃO JOSÉ DO RIO CLARO - MT
}

\section{CONSUMPTION OF CONTRACEPTIVE METHODS BY THE POPULATION OF SÃO JOSÉ DO RIO CLARO - MT}

Durante,Jaqueline'; Alcântara, Anelise Montañes²; Zagonel, Ivete Palmira Sanson ${ }^{3}$

\begin{abstract}
Farmacêutica. Aluna da especialização em Farmacologia e Dispensação Farmacêutica do Instituto Brasil de Pós-Graduação-IBRAS.

${ }^{2}$ Farmacêutica. Mestre em Biotecnologia aplicada à Saúde da Criança e do Adolescente da Faculdades Pequeno Príncipe-FPP. Orientadora.

${ }^{3}$ Enfermeira. Docente do Programa de Pós-Graduação em Biotecnologia aplicada à Saúde da Criança e do Adolescente da FPP. Co-orientadora.
\end{abstract}

\section{RESUMO:}

O presente artigo teve como objetivo geral identificar padrões de consumo dos métodos contraceptivos utilizados pela população do município de São José do Rio Claro-MT e como objetivos específicos: conhecer o perfil sócio-demográfico da população em estudo, verificar o conhecimento dos métodos contraceptivos pela população, examinar o local de aquisição, a indicação e os motivos de escolha dos métodos contraceptivos, além de verificar a adequação do uso de anticoncepcionais orais e injetáveis. Como método foi utilizado uma pesquisa descritiva de natureza qualitativa, desenvolvida entre dezembro de 2009 a janeiro de 2010 por meio de entrevistas domiciliares no município de São José do Rio Claro-MT. A amostra foi composta por 50 sujeitos com faixa etária entre 18 a 57 anos, de ambos os sexos, selecionados aleatoriamente, estando a participação de cada entrevistado sujeita a um consentimento livre e esclarecido. Os resultados apontaram que a maioria dos entrevistados era jovem (54\% entre 18 a 27 anos), do sexo feminino (82\%), católicos $(84 \%)$, casados $(50 \%)$ e com filhos $(58 \%)$. Observou-se ainda que o consumo de $80 \%$ dos sujeitos da pesquisa se concentra em três dos métodos contraceptivos: anticoncepcional oral $(46 \%)$, preservativo $(26 \%)$ e laqueadura tubária $(18 \%)$, sendo que $10 \%$ deles utilizam em associação o anticoncepcional oral e o preservativo. Concluiu-se que a maioria das mulheres consumidoras de anticoncepcionais orais e injetáveis relata seguir orientação médica e utilizar corretamente o método. Os fatores que mais influenciam na escolha do método contraceptivo são praticidade, confiança, segurança, comodidade e preço.

Palavras-chave: anticoncepção; farmacoepidemiologia; uso de medicamentos.

\section{ABSTRACT:}

This study had as main purpose to identify patterns of consumption of contraceptive methods used by the population of São José do Rio Claro-MT and as specific objectives: know the socio-demographic profile of the study population, evaluate the knowledge about contraceptive methods by population, examine the place of purchase, the indications and reasons for choosing contraceptive methods, and also verify the suitability of using oral contraceptives and injectables. The method was a qualitative and descriptive research, carried out between December 2009 and January 2010 through interviews in the municipality of Sao Jose do Rio Claro-MT. The sample comprised 50 
subjects aged between 18 to 57 years, of both genders, randomly selected, with the participation of each respondent subject done under free and informed consent form. The results showed that most respondents were young (54\% from 18 to 27 years), female $(82 \%)$, Catholics $(84 \%)$, married $(50 \%)$ with children $(58 \%)$. It has been also observed that the consumption of $80 \%$ of the subjects of research focuses on three methods of contraception: oral contraceptives (46\%), condoms (26\%) and tubal ligation $(18 \%)$, while $10 \%$ of them use an association of oral contraceptives and condoms. It was concluded that most women consuming oral or injectable contraceptives report they follow medical advice and use the method correctly. The factors that influence the most the choice of contraceptive method are practicality, reliability, safety, convenience and price.

KEYWORDS: contraceptive methods, pharmacoepidemiology, use of medications.

\section{INTRODUÇÃO:}

A escolha de um método contraceptivo deve levar em consideração aspectos como a idade, a plena aceitação por parte do casal, a eficiência, a facilidade de utilização, o grau de inocuidade, a reversibilidade ao suspender o uso, os fatores sócioeconômicos e a religião (MARIANI, 2009). Essa escolha deve contar com informações adequadas, envolvendo um planejamento familiar apropriado, ficando o usuário livre para escolher e utilizar o melhor método para si ou para o casal. Para o alcance de uma escolha eficaz e de qualidade, além do conhecimento do corpo, é necessário saber quais são os diferentes métodos disponíveis no mercado farmacêutico, e como ter acesso a informações junto aos profissionais da saúde e aos locais de saúde que oferecem estes métodos (PINOTTI; FONSECA, 2006).

Não existe um método contraceptivo melhor que o outro, cada um apresenta vantagens e desvantagens. Da mesma forma, também não existe um método $100 \%$ eficaz, todos têm uma probabilidade de falha. Assim, um método contraceptivo pode ser adequado para uma pessoa e não ser apropriado para outra (BRASIL, 2006).

Nesse sentido, o presente artigo procura dissertar sobre os Estudos de Utilização de Medicamentos (EUM), um campo de estudo da Farmacoepidemiologia, e versar sobre o consumo dos métodos contraceptivos pela população do município de São José do Rio Claro-MT.

A Farmacoepidemiologia se subdivide em dois grandes grupos: os Estudos de Utilização de Medicamentos (EUMs) e a Farmacovigilância. Estas duas atividades têm como objetivo conhecer, analisar e avaliar o impacto dos medicamentos sobre as populações humanas (STORPIRTIS et al., 2008). Os EUMs se destinam ao acompanhamento da comercialização, distribuição, prescrição e uso de medicamentos em uma sociedade, com principal enfoque em suas conseqüências sanitárias, sociais e econômicas resultantes (LAPORTE apud STORPIRTIS et al., 2008). São ferramentas valiosas para acompanhar o uso de medicamentos no decorrer do tempo, identificar 
problemas potenciais associados a seu uso e avaliar os efeitos de intervenções educativas. Os EUMs representam apoios decisivos na elaboração de políticas governamentais no campo da saúde e abrange diferentes temas de investigação relacionados ao uso de medicamentos (CASTRO apud STORPIRTIS et al., 2008).

Conforme Gomes e Reis (2001) as prescrições são avaliadas para que haja uma redução da quantidade e da intensidade dos efeitos indesejáveis causados por problemas relacionados ao uso dos medicamentos, reconhecendo que a prática de uso de medicamentos não se limita apenas aos fatores farmacoterapêuticos, procurando demonstrar os padrões coletivos desse consumo. Desse modo, a Farmacoepidemiologia é útil na provisão de informações sobre os efeitos benéficos e indesejados de qualquer fármaco, permitindo uma melhor compreensão da relação risco-benefício para o uso racional dos medicamentos (PEREIRAet al., 2008).

Um exemplo desses efeitos indesejáveis é a interação medicamentosa dos anticoncepcionais hormonais, principalmente dos que contém estrógeno sintético, com o tabaco utilizado por mulheres acima dos 35 anos ou que tenham algum fator que predisponha à trombose. O hábito de fumar aumenta o risco de graves efeitos adversos cardiovasculares. Outro exemplo de interação medicamentosa com os anticoncepcionais hormonais é o uso concomitante com antibióticos ou anticonvulsivantes, que podem provocar diminuição da eficácia do método contraceptivo (KOROLKOVAS, 2008).

\section{OBJETIVOS}

A pesquisa teve como objetivo geral identificar padrões de consumo dos métodos contraceptivos utilizados pela população do município de São José do Rio Claro-MT e como objetivos específicos conhecer o perfil sócio-demográfico da população em estudo, verificar o conhecimento dos métodos contraceptivos pela população, examinar o local de aquisição, a indicação e os motivos de escolha dos métodos contraceptivos, envolvendo a prática religiosa, além de verificar a adequação do uso de anticoncepcionais orais e injetáveis.

\section{MÉTODOS}

O estudo foi realizado no município de São José do Rio Claro-MT por meio de visitas domiciliares. Trata-se de uma pesquisa descritiva de natureza qualitativa desenvolvida entre dezembro de 2009 e janeiro de 2010 tendo como instrumento de coleta de informações a entrevista semi-estruturada composta por padrões de consumo dos métodos contraceptivos utilizados pela população do município, dados sócio-demográficos, conhecimento dos métodos contraceptivos pela população, local 
de aquisição, indicação e os motivos de escolha dos métodos contraceptivos envolvendo a prática religiosa; e adequação do uso de anticoncepcionais hormonais.

As entrevistas foram gravadas e posteriormente transcritas respeitando as respostas dadas pelos entrevistados. A análise qualitativa foi feita de acordo com a técnica de análise de conteúdo temática proposta por Minayo (2007), que possibilitou desvelar o sentido a partir dos depoimentos, as significações do fenômeno em estudo, e conhecer como são utilizados e escolhidos os métodos anticoncepcionais. Foram realizadas 50 entrevistas em residências selecionadas aleatoriamente, com pessoas na faixa etária entre 18 a 57 anos com vida sexual ativa, de ambos os sexos, que residiam em São José do Rio Claro-MT e que concordaram em participar da pesquisa. No total, 4 pessoas se recusaram a participar da pesquisa.

A pesquisa seguiu os preceitos éticos da Resolução n 196 de outubro de 1996 do Conselho Nacional de Saúde. Os entrevistados foram informados sobre o anonimato, o sigilo das informações e a respeito da eventual desistência. O projeto de pesquisa foi aprovado pelo Comitê de Ética da Faculdade Cathedral sob parecer 042/2009.

\section{RESULTADOS E DISCUSSÕES}

Com relação aos dados sócio-demográficos identificados foi possível perceber que a maioria dos entrevistados é jovem (54\% entre 18 a 27 anos), do sexo feminino $(82 \%)$, católico $(84 \%)$, casado $(50 \%)$, com filhos (58\%). Dentre os que tinham filhos, o número máximo era de 7 filhos, com uma média de 2 filhos. A média nacional de filhos segundo resultados da Pesquisa Nacional por Amostra Domicílios (PNAD) de 2007, analisados pelo Instituto de Pesquisa Econômica Aplicada (IPEA), demonstra que a mulher brasileira continua optando por ter poucos filhos. A média em 2007 era de 1,8 filhos/mulher, enquanto na década de 60 a média era de 6 filhos (ABE, 2007). Portanto, a média de filhos da amostra pesquisada em São José do Rio Claro-MT se encontra próxima aos parâmetros nacionais.

Dos solteiros (38\%), 24\% declararam ter um(a) parceiro(a) fixo(a). Quanto à escolaridade, $26 \%$ não haviam concluído o ensino fundamental, $38 \%$ haviam completado o ensino médio, $16 \%$ tinham ensino superior incompleto e $14 \%$ ensino superior completo.

Todos os entrevistados declararam não ser fumantes, e apenas 1 deles era exfumante. Com relação a bebidas alcoólicas, $30 \%$ declararam nunca fazer uso, $60 \%$ às vezes, e $10 \%$ fazer uso quase sempre ou sempre.

A Tabela 1 mostra a distribuição de utilização dos métodos contraceptivos. Observou-se que $80 \%$ dos sujeitos da pesquisa consomem com maior prevalência cerca de três métodos contraceptivos: anticoncepcional oral (46\%), preservativo (26\%) 
e laqueadura tubária (18\%), sendo que 10\% deles utilizam anticoncepcional oral e preservativo e $4 \%$ deles pílula do dia seguinte e preservativo.

É importante considerar que nenhum dos métodos contraceptivos, exceto o preservativo, oferece proteção contra doenças sexualmente transmissíveis (DSTs). Desta forma, os usuários devem associar o método de sua escolha com o preservativo masculino ou feminino para reduzir o risco de doenças. O preservativo pode também ser utilizado como uma segunda barreira anticoncepcional visando o aumento da eficácia, além de atuar como método secundário no caso falha na administração do medicamento oral.

Outra forma de associação utilizada é a pílula do dia seguinte com o preservativo, o que diminui o risco de gravidez, tomando a mesma até 72 horas após o ato sexual. A pílula do dia seguinte utiliza compostos hormonais concentrados e por um curto período de tempo nos dias posteriores a relação sexual diferindo dos demais métodos que são utilizados antes e durante a relação. Esta pílula pode ser usada caso aconteça algum incidente, como estouro do preservativo ou estupro, porque o uso freqüente pode ser extremamente prejudicial ao organismo, provocando alterações menstruais e gerando problemas como náuseas, enjôos ou vômitos. Dessa forma a pílula do dia seguinte não deve ser usada de forma planejada, previamente programada, ou substituir métodos contraceptivos de rotina (BRASIL, 2010).

TABELA 1 - Distribuição da amostra de acordo com a utilização dos métodos contraceptivos.

\begin{tabular}{lc}
\hline \multicolumn{1}{c}{ Método contraceptivo } & Porcentagem \\
\hline Anticoncepcional oral & $46 \%$ \\
Preservativo & $26 \%$ \\
Laqueadura tubária & $18 \%$ \\
Vasectomia & $6 \%$ \\
Pílula do dia seguinte & $4 \%$ \\
Anticoncepcional Injetável & $4 \%$ \\
\hline
\end{tabular}

Fonte: Dados coletados durante a pesquisa de campo em São José do Rio Claro - MT, 2009 e 2010.

${ }^{*}$ A soma ultrapassa $100 \%$ pois a questão permitia mais de uma resposta.

O anticoncepcional oral é o método mais utilizado e o mais aceito entre as mulheres, pois além de ser facilmente encontrado nas farmácias comunitárias, a política governamental distribui gratuitamente este anticonceptivo nas unidades de saúde. O preservativo, outro método consumido pelos usuários, também é distribuído na rede pública gratuitamente. Esses dois métodos citados e o anticoncepcional injetável também são encontrados na farmácia popular ou em drogarias e farmácias privadas credenciadas no programa do Ministério da Saúde, que chega a dar $90 \%$ de 
desconto para população na compra desses medicamentos de uso contínuo. De acordo com Scarazzati (2007) para adquirir esse medicamento o indivíduo tem que ter em mãos uma receita médica e o CPF da usuária, obtendo assim os descontos indicados para cada medicamento. O município de São José do Rio Claro não possui o programa Farmácia Popular e nem farmácias e drogarias credenciadas ao governo.

Verificou-se que o uso de anticoncepcional oral diminui com a idade, enquanto o uso de outros métodos aumenta com o decorrer do tempo. Como exemplos de métodos que são mais usados com avanço da idade, pode-se citar a vasectomia e a laqueadura tubária. Todos os homens entrevistados que afirmaram ser vasectomizados eram casados e tinham idade mínima de 43 anos. Todas as mulheres do estudo com laqueadura tubária tinham pelo menos dois filhos, estavam casadas e com a idade mínima de 31 anos.

A esterilização de mulheres, processo bastante difundido na sociedade brasileira, é um importante fator de infertilidade feminina. Cerca de uma em cada três mulheres que fazem a laqueadura se arrepende após a opção por este método. Como esse fato não pode ser desprezado, é importante que os critérios de inclusão destas mulheres sejam rigorosos. Levando-se em consideração a existência de outros métodos contraceptivos temporários, os definitivos deverão ser a última opção, pois gerarão a infertilidade definitiva (MACEDO apud SCHRAMM, 2005).

Conforme Costa apud Schramm (2005, p.171) a preferência pela esterilização no Brasil não é uma escolha, e sim falta de opção como pode ser verificado abaixo:

\begin{abstract}
Consolidou-se de forma perversa uma cultura reprodutiva onde, ainda muito jovens, as mulheres, por desinformação e ausência de outras alternativas, incluem em seu projeto de vida a cesariana e a esterilização. Por esta opção pagam caro, pois além da mortalidade referida herdam seqüelas quase sempre definitivas, aumento da mortalidade perinatal e altas e inconcebíveis taxas de arrependimento pós-laqueadura. Os estudos dedicados ao arrependimento pós-laqueadura estabelecem uma relação direta entre esta situação e a desinformação sobre a existência e disponibilidade de outras alternativas contraceptivas, bem como a reversibilidade do procedimento cirúrgico. Esse significativo percentual, as esterilizações são realizadas no curso das cesarianas, freqüentemente indicadas com o objetivo da realização simultânea da laqueadura. As altas taxas de cesariana que o Brasil exibe estão entre as mais elevadas do mundo (COSTA apud SCHRAMM, 2005, p.171).
\end{abstract}

Em relação a quem indicou o método contraceptivo, verificou-se que em $44 \%$ dos casos o médico foi responsável pela indicação, em $20 \%$ foi escolhido por conta própria, $14 \%$ por indicação de amigos ou parentes, $8 \%$ a partir de sugestão de pessoas na escola, e em $8 \%$ a partir da orientação de farmacêutico ou balconista (Tabela 2). 
TABELA 2 - Distribuição da amostra de acordo com quem fez a indicação do método contraceptivo.

Quem indicou

\section{Médico}

Conta própria*

Amigos ou parentes

Escola

Farmacêutico ou balconista

\section{Porcentagem}

$44 \%$

$20 \%$

$14 \%$

$8 \%$

$8 \%$

Fonte: Dados coletados durante a pesquisa de campo em São José do Rio Claro - MT, 2009 e 2010.

*Pode ser entendido como automedicação ou decisão livre sobre a escolha de métodos definitivos.

Também se relacionou o método contraceptivo de acordo com sua indicação. Desta maneira, verificou-se que os anticoncepcionais orais e a vasectomia foram preferencialmente indicados por médicos, que a opção pela ligadura tubária foi por conta própria e que a utilização de preservativos teve influência da escola, parentes, médicos, farmacêuticos entre outros.

Para iniciar a utilização de um método contraceptivo é necessário compreender o seu funcionamento. Portanto, os profissionais da saúde, incluindo o farmacêutico, devem orientar as pessoas sobre a utilização adequada do método, bem como considerar fatores como a idade do paciente, seu estilo de vida, se tem ou pretende ter mais filhos, seu estado da saúde em geral e a necessidade de proteção contra infecções de transmissão sexual. Vale ressaltar que antes de optar por um método contraceptivo específico, é recomendável que se consulte um médico, de preferência um ginecologista, que será capaz de avaliar cada caso, já que nem todas as mulheres se adaptam a todos os métodos disponíveis.

É importante considerar que algumas pessoas praticam a automedicação, ou seja, começam a usar algum método contraceptivo por conta própria sem recomendação médica, por influência de amigos, colegas e familiares. Uma situação que ocorre principalmente com adolescentes que estão iniciando a vida sexual é a falta de diálogo, a vergonha, o receio de comentar ou até mesmo de sanar dúvidas comuns com os próprios pais, e acabam optando por escolhas que podem ocasionar riscos de gravidez ou efeitos colaterais que podem ser graves à saúde (ZANDONA, 2009).

Outra situação muito freqüente são pessoas que procuram os farmacêuticos e até mesmos os balconistas para indicarem um contraceptivo. Esta prática também é inadequada, pois a indicação de um anticoncepcional hormonal oral ou injetável sem prescrição médica pode incentivar o usuário à automedicação. $O$ farmacêutico só pode fazer a automedicação responsável com medicamentos isentos de prescrição médica. 
Já no caso dos métodos de barreira, como os preservativos masculinos e femininos, esses profissionais e até mesmo as escolas devem incentivar o seu uso não apenas para evitar gravidez, mas também como proteção contra as doenças sexualmente transmissíveis.

Quanto ao local de acesso aos métodos contraceptivos identificados, a farmácia destaca-se como a principal fornecedora dos métodos, por ser um lugar de fácil acesso. Em relação aos métodos definitivos como a laqueadura e a vasectomia, foram realizados em maior freqüência em hospital particular. Esses procedimentos definitivos foram regulamentados pela Lei Federal $n^{\circ} 9.263 / 96$, que garante o direito à cirurgia pelo SUS de homens e mulheres com capacidade civil plena e maiores de 25 anos de idade ou pelo menos com dois filhos vivos (BRASIL, 1996) (Tabela 3).

TABELA 3 - Distribuição da amostra de acordo com o local de acesso aos métodos contraceptivos.

\begin{tabular}{lc}
\hline \multicolumn{1}{c}{ Local } & Porcentagem \\
\hline Farmácia & $62 \%$ \\
Hospital (Particular) & $6 \%$ \\
Unidade de Saúde & $6 \%$ \\
Hospital (SUS) & $2 \%$ \\
Supermercado & $2 \%$ \\
\hline
\end{tabular}

Fonte: Dados coletados durante a pesquisa de campo em São José do Rio Claro-MT, 2009 e 2010.

Outro local de acesso citado na pesquisa foi o supermercado, mas apenas para compra de preservativo, uma vez que tais estabelecimentos são proibidos de vender qualquer tipo de medicamentos, mesmo os isentos de receita médica (CAMPOS 2004).

Em relação à correta utilização do anticoncepcional oral e injetável, a maioria $(61,5 \%)$ das mulheres usa corretamente o método, $11,5 \%$ utiliza de forma inadequada e cerca de $27 \%$ não informaram a forma de utilização. Para que o método seja eficaz na prevenção da gravidez, deve-se usá-lo adequadamente. No caso do anticoncepcional oral, é necessário tomar o comprimido diariamente sempre no mesmo horário, não esquecendo nenhum comprimido.

$\mathrm{Na}$ análise qualitativa, alguns relatos demonstram a falta de orientação das usuárias, pois as pacientes faziam uso inadequado do medicamento.

\footnotetext{
"Quando eu usava tomei quando comprei, não esperei o $1^{\circ}$ dia da menstruação"(E1, 20 anos).

"Quando tomava, a menstruação descia, ai tomava no quinto dia" (E13, 51 anos).
} 
Como fatores que influenciam na decisão de optar pelo uso de anticoncepcional oral foram citados a praticidade, a confiança, segurança, comodidade, preço, medo de injeção, para regular a menstruação e começo de namoro.

\footnotetext{
"Sim uso pílula, é mais pratico e confiável"(E16, 28 anos).

"Uso sim comprimido, é o método que me sinto mais segura"(E20, 25 anos).

"A minha mulher usa pilula Ciclo 21, método mais seguro, gasta menos com esse método, porque usa só este"(E24, 23 anos).

"Sim uso pílula mais comodidade, minha igreja não influencia" (E25, 29 anos).

"Sim tomo pílula contínua Adoless, tenho medo de injeção e porque esqueço de toma [comprimidos]"(E30, 23 anos).

"Sim, pílula. Para regular menstruação e porque eu comecei namora, aí não quero filho agora. Quero evitargravidez"(E47, 22 anos).
}

A opção pelo anticoncepcional hormonal injetável se dá pelo risco de esquecimento de tomar os comprimidos, pela eficácia e pelo preço.

"Sim, injeção, porque esqueço o comprimido e porque é eficaz"(E48, 19 anos)

"Sim, injeção, porque esquecia muito comprimido, e prefiro injeção até pelo preço, acho mais barato que comprimido"(E 50, 28 anos).

Os motivos ligados à escolha pela laqueadura tubária foram não querer mais filhos, praticidade, risco de esquecer o comprimido, não adaptação a outros métodos e problemas de saúde.

"Fiz laqueadura, não queria ter mais filhos, por causa da situação financeira. Fiz porvontade própria"(E12, 35 anos).

"Fiz laqueadura, não queria ter mais filhos, tive que faze porque quando tomava pílula dava dor de cabeça, inchava barriga, mesmo tomando ao dormir, tentei usar injeção mais não deu certo, ai após 3 filhos optei por laqueadura, se não teria mais uns 3 filhos"(E42, 34 anos).

"Sim, laqueadura, por problemas de saúde. É que aos 3 meses de gestação de todas as gravidez, a placenta descola, ai eu tomava remédio como o Daquitil, pra segura e ficava de repouso. Até na ultima gestação o médico queria que eu tirasse, mas eu não quis porque era a única menina que vinha, ai não deixei e fiquei a gravidez inteira na cama, mas consegui ter a menina"(E46, 34 anos).

"Sim, fiz laqueadura, porque já tive 7 filhos, tá bom, não queria mais e também por que quando tomava comprimido esquecia, se não tivesse feito teria mais uns"(E49, 58 anos).

Entre os motivos da opção do homem pela vasectomia identificados nas falas, está a intenção de facilitar a vida da companheira, preocupação com a saúde da companheira, e livrá-la de tomar os comprimidos. 
"Sim, fiz vasectomia para preservar a saúde da minha esposa, pois ela não se dava bem com anticoncepcional"(E22, 46 anos).

"Sim, fiz vasectomia, por ser mais fácil, minha esposa não precisa tomar mais remédio, e pela situação financeira”(E37, 43 anos).

Os motivos referentes ao uso do preservativo são: agilidade, praticidade, ausência de parceira fixa, segurança, prevenção de doenças sexualmente transmissíveis e risco de esquecer comprimidos.

\footnotetext{
“Uso preservativo pela facilidade, agilidade, e por não ter parceira fixa” (E6, 24 anos).

"Uso camisinha, pra não adquirir doenças" (E7, 23 anos).

"Uso camisinha, por segurança, não quero ter mais filhos" (E15, 27 anos).

"Uso camisinha, mais seguro, porque não tenho parceira fixa" (E17, 22 anos).

"Sim, uso camisinha, acho mais seguro" (E28, 43 anos).
}

Nos discursos dos sujeitos da pesquisa, a escolha pelos métodos associados está relacionada à prevenção de doenças sexualmente transmissíveis, à segurança, preferência, ao esquecimento de comprimidos e à confiança. A pílula do dia seguinte deve ser usada apenas emergencialmente, isto é, em caso de violência sexual, ou falhas, como: rompimento de preservativo, deslocamento de diafragma, esquecimento da pílula comum por dois dias seguidos ou mais. A pílula do dia seguinte também pode ser usada em situações onde houve relação sem uso de método contraceptivo (LEFEFRE, 2010).

Tomo comprimido e uso camisinha, para evitar doenças transmissíveis (E4, 18 anos).

Utilizo pílula e preservativo, segurança total (E8, 23 anos).

Sim, uso anticoncepcional e camisinha. Camisinha mais segurança e anticoncepcional pela eficácia (E11,23 anos).

Sim, minha mulher usa pilula e a camisinha, é mais seguro, por preferência, opção nossa (E18, 22 anos).

Uso camisinha sempre porque ela esquece alguns comprimidos (E18, 22 anos).

Uso preservativo e pílula do dia seguinte, mais seguro e confiável (E10, 19 anos).

Em alguns casos, a não utilização de método contraceptivo está relacionada ao desejo de ser mãe ou à fase pós-menopausa.

“Não tomo nenhum [comprimido], estou querendo engravidar" (E5, 22 anos). 
"Não estou tomando [comprimido], quero ter mais um filho"(E45, 34 anos).

"Usei comprimido até meus 50 anos, agora estou na menopausa, uso Klimater. Não quis ter mais filhos fiquei só com uma filha, me acomodei, passou os anos, e por causa do dinheiro"(E13, 51 anos).

Como outros motivos apreendidos nas falas para a não utilização de um método anticoncepcional, estão a religião, achar-se muito nova para iniciar algum método ou ter parceiro que já optou pela vasectomia.

"Não uso nada [nenhum método]. Sou muito nova"(E2, 18 anos).

"Não uso, não tenho filhos por opção, meu esposo fez vasectomia, vida de pastor é corrida, não tem tempo"(E14, 43 anos).

"Já usei Ciclo 21 meu corpo se adaptou melhor, hoje não uso por que a religião não permite"(E1, 20 anos).

Com relação ao conhecimento dos entrevistados sobre métodos contraceptivos distintos do que declaravam utilizar, $64 \%$ citaram o preservativo masculino, $52 \%$ o anticoncepcional injetável, $52 \%$ o anticoncepcional oral, $48 \%$ o DIU (dispositivo intra-uterino), $32 \%$ a tabelinha, $22 \%$ o diafragma, $16 \%$ o adesivo, $6 \%$ o coito interrompido, $4 \%$ a pílula do dia seguinte, $4 \%$ o preservativo feminino, $2 \%$ o anel vaginal, $2 \%$ a laqueadura tubária e $2 \%$ a vasectomia (Tabela 5 ).

TABELA 5 - Distribuição da amostra de acordo com os métodos contraceptivos conhecidos.

\begin{tabular}{lc}
\hline \multicolumn{1}{c}{ Métodos contraceptivos } & Porcentagem \\
\hline Preservativo masculino & $64 \%$ \\
Anticoncepcional Injetável & $52 \%$ \\
Anticoncepcional oral & $52 \%$ \\
DIU & $48 \%$ \\
Tabelinha & $32 \%$ \\
Diafragma & $22 \%$ \\
Adesivo & $16 \%$ \\
Coito interrompido & $6 \%$ \\
Pílula do dia seguinte & $4 \%$ \\
Preservativo feminino & $4 \%$ \\
Anel vaginal & $2 \%$ \\
Laqueadura tubária & $2 \%$ \\
Vasectomia & $2 \%$ \\
\hline
\end{tabular}

Fonte: Dados coletados durante a pesquisa de campo em São José do Rio Claro - MT, 2009 e 2010.

${ }^{*}$ A soma ultrapassa $100 \%$ pois a questão permitia mais de uma resposta. 
Mesmo conhecendo outros métodos como o DIU, o diafragma, o adesivo e o anel vaginal, a população de São José do Rio Claro-MT não os utiliza por ser de difícil acesso, pois precisam se locomover para municípios maiores nos quais existem médicos especialistas para orientar, poder implantar e fazer a manutenção dos mesmos. No município pesquisado, não há médico ginecologista. Outro aspecto importante a ser citado são os preços que não são acessíveis à maioria da população, além da falta de conhecimento. Alguns até sabem que esses métodos existem, mas não conhecem as vantagens nem sabem como é colocado e como funciona no organismo.

\section{CONCLUSÕES}

No estudo dos padrões de consumo dos métodos contraceptivos por meio de análises qualitativas foi identificado que a grande maioria das mulheres consumidoras de anticoncepcionais hormonais seguiu orientação médica e utiliza corretamente o método contraceptivo indicado, embora haja ocorrência de mau uso de anticoncepcional oral. Os fatores que mais influenciam na escolha do método contraceptivo são praticidade, confiança, segurança, comodidade e preço.

O uso inadequado de métodos contraceptivos pode ocasionar transtornos à mulher e à família, pois está exposta a ocorrência da gravidez, efeitos colaterais indesejados, vulnerabilidade a doenças sexualmente transmissíveis. Somente com a apropriação consciente das vantagens que a educação em saúde fornece é que a usuária e por extensão sua família, se beneficiarão. O planejamento familiar extrapola a dimensão apenas reprodutiva, pois envolve questões sociais, culturais, religiosas que precisam ser consideradas no momento da prescrição, dispensação e uso de contraceptivos.

O estudo procura oferecer subsídios necessários a correção de eventuais distorções quanto à utilização de métodos contraceptivos. Neste contexto é importante destacar a importância que o profissional farmacêutico desempenha na dispensação e na educação à saúde, orientando o usuário sobre o uso correto dos medicamentos. Destaca-se ainda, que pela proximidade que o farmacêutico tem com a população, este profissional desempenha um papel crucial na orientação dos usuários para a promoção do uso racional de medicamentos.

\section{REFERÊNCIAS}

ABE. Érica. Média de filhos de brasileira é 1,8 mulher, mostra analise do IPEA. Brasília. 2008. Disponível em http://g1.globo.com/noticias/brasil. Acesso dia: 16/01/2010. 
AMABIS, Jose Mariano; MARTHO, Gilberto Rodrigues. Biologia: biologia das células. 2. ed. São Paulo: Moderna, v.1, 2004.

BRASIL. Ministério da Saúde. Lei no 9263 de 12 de janeiro de 1996. Trata do planejamento familiar, estabelece penalidades e dá outras providências.

BRASIL. Ministério da Saúde. Direitos sexuais, direitos reprodutivos e métodos anticoncepcionais. Brasília: Ministério da Saúde, 2006.

BRASIL. Sistema Nacional de Auditoria. Tire suas dúvidas sobre a pílula do dia seguinte. Disponível em http://sna.saude.gov.br/imprimir.cfm?id=4167. Acesso dia: 03/02/2010.

CAMPOS, Shirley. Supermercados não podem vender remédios. 2004. Disponível em: http://www.drashirleydecampos.com.br/noticias/13047. Acesso dia: 16/01/2010. FACHIN, Odília. Fundamentos de metodologia. 5 Ed. São Paulo: Saraiva, 2006.

GOMES, Maria José Vasconcelos de Magalhães; REIS, Adriano Max Moreira. Ciências Farmacêuticas. Uma abordagem em farmácia hospitalar. São Paulo: Atheneu, 2001.

KOROLKOVAS, Andrejus; FRANÇA, Francisco Faustino de Albuquerque Carneiro. Dicionário Terapêutico Guanabara. Rio de Janeiro: Guanabara Koogan, 2008.

LEFEVRE, Fernando (org.). O profissional de saúde, o adolescente e a contracepção de emergência: ajudando a decidir: guia de orientação para o profissional de saúde. Instituto de Pesquisas do Discurso do Sujeito Coletivo, 2010.

LOPES, Sonia; ROSSO, Sergio. Biologia: volume único. São Paulo: Saraiva, 2005.

MARIANI, Mirella. Métodos anticoncepcionais. 2009. Disponível em: www.reprodusite.hpg.ig.com.br/metodos.htm. Acesso dia 26/10/2009.

MINAYO, Maria Cecília Souza. O desafio do conhecimento: pesquisa qualitativa em saúde. 10 ed. São Paulo: HUCITEC, 2007.

PETTA, Carlos Alberto; FAÚNDES, Anibal. Métodos anticoncepcionais. São Paulo: Contexto, 1998.

PEREIRA, G. J da S. Estudo de utilização de medicamentos na clínica médica. Revista Brasileira Farmacêutica. v. 89. Paraíba, 2008. Disponível em: 
http://www.revbrasfarm.org.br/pdf/2008/RBF_R3_2008/155_pag_267a271_estudo_ut ilizacao.pdf. Acesso dia 10/11/2009.

PINOTTI, José Aristodemo; FONSECA, Angela Maggio. Saúde da mulher. São Paulo: Contexto, 2006.

REIS, Alberto Olavo Advincula; RIBEIRO, Maria Aparecida Andrés. Métodos anticoncepcionais. 2007. Disponível em: http://www.fozdoiguacu.pr.gov.br/noticias/ link55.htm Acesso dia 12/10/2009.

SCARAZZATI, Luciane. Anticoncepcional com desconto exige receita. São Paulo. 2007. Disponível em http://g1.globo.com/noticias/brasil/o,,mul43938-5598,00anticoncepcional+com+desconto+exige+receita.html. Acesso dia 20/01/2010.

SCHRAMM, Fermin Roland; BRAZ, Marlene (Org.). Bioética e saúde: novos tempos para mulheres e crianças? Rio de Janeiro: Fiocruz, 2005.

SOUZA, José Bento. Mulher e contracepção: evolução e conquista. São Paulo: Alaúde, 2003.

STORPIRTIS, Silvia; MORI, Ana Luiza Pereira Moreira; YOCHIY, Angélica; RIBEIRO, Eliane; PORTA, Valentina. Farmacologia básica \& clínica. Rio de Janeiro: Guanabara Koogan, 2008.

ZANDONA, Letícia. Tipos de pílula anticoncepcional. 2009. Disponível em: http://www.semprefeminina.com.br/tag/pilula-anticoncepcional/. Acesso dia 15/01/2010. 